\title{
Análise do Impacto do Estágio Supervisionado sobre o Desempenho da Policlínica Odontológica Central da Brigada Militar: enfoque em resultados
}

\author{
Analysis of the Impact of Supervised Internship on the Performance of the Dental Central Polyclinic \\ of Brigada Militar: focus on results
}

Maximiliano Schünke Gomes ${ }^{1}$, Regis Reche ${ }^{2}$, Fernando Neves Hugo ${ }^{3}$

\section{Abstract}

The aim of this study was to analyze the impact of supervised internship (ES) of students from FO-UFRGS, focusing on results, on the performance of the Dental Central Polyclinic (POC) of Brigada Militar (BM) in the period between April-2008 and December-2009. To do so, a monthly survey of the number of treated patients (PA) and the number of dental procedures (PO) performed by trainees (E) and dentists (CD) of the service during the period was conducted. From the comparison of PA and PO performed by $E$ and $\mathrm{CD}$, productivity indicators of the ES (IPE) for PA (IPE-pa) and PO (IPE-po) were proposed and calculated, as well as an indicator of overall productivity of the ES (IPE-g). The results showed average values of IPE-pa $=1.63( \pm 0.74)$, IPE-po $=1.48( \pm 0.49)$ and IPE-g $=1.55( \pm 0.57)$, indicating that the ES provided a general increase in productivity equivalent to more than one and a half (1.5) CD per month for the service of POC, showing average monthly increase of $10.46 \%$ in the overall productivity of the service.

Key-words: Dental education; Dental health services; Employee performance appraisal; Health human resource evaluation; Organizational efficiency.

\section{Resumo}

O objetivo deste estudo foi analisar o impacto do estágio supervisionado (ES) de alunos da FO-UFRGS, com enfoque nos resultados, no desempenho da Policlínica Odontológica Central (POC) da Brigada Militar (BM) no período de abril de 2008 a dezembro de 2009. Para tanto, foi realizado um levantamento mensal do número de pacientes atendidos (PA) e do número de procedimentos odontológicos $(P O)$ realizados por estagiários $(E)$ e por cirurgiões-dentistas (CD) do serviço no período estudado. A partir da comparação de $P A$ e $P O$ realizados por $E$ e $C D$, foram criados indicadores de produtividade do ES (IPE) em relação a PA (IPE-pa) e PO (IPE-po), bem como um indicador de produtividade geral do ES (IPE-g). Os resultados demonstraram médias de IPE$\mathrm{pa}=1,63( \pm 0,74)$, IPE-po $=1,48( \pm 0,49)$ e IPE-g = 1,55 $( \pm 0,57)$, indicando que o ES proporcionou acréscimo de produtividade geral equivalente a mais de um e meio $(1,5) \mathrm{CD}$ por mês para o serviço da POC, revelando aumento médio mensal de $10,46 \%$ na produtividade geral do serviço.

Palavras-chave (BVS): Educação em Odontologia; Serviços de saúde bucal; Avaliação de desempenho; Avaliação de recursos humanos em saúde; Eficiência organizacional.
${ }^{1}$ Capitão do Quadro de Oficiais Especialistas em Saúde da Brigada Militar do Rio Grande do Sul; Especialista em Gestão em Saúde (UFRGS); Especialista e Mestre em Endodontia (ULBRA); Doutorando em Odontologia (UFRGS)

${ }^{2}$ Capitão do Quadro de Oficiais Especialistas em Saúde da Brigada Militar do Rio Grande do Sul; Especialista em Ortodontia (ABO-PR)

${ }^{3}$ Professor Adjunto do Departamento de Odontologia Preventiva e Social (da Universidade Federal do Rio Grande do Sul; Professor Permanente do Programa de Pós-Graduação em Odontologia da Faculdade de Odontologia da UFRGS; Doutor em Odontologia (UNICAMP)

\section{Correspondência: Maximiliano Schünke Gomes}

Endereço: Rua Sete de Setembro, 372 - CEP 90010-190, Porto Alegre - RS Brasil

Fone: (51) 3288-2968 / (51) 9956-9626

E-mail: endomax@bm.rs.gov.br

Data de Submissão: 09/10/2010

Data de Aceite: 23/11/2010

\section{Introdução}

A Brigada Militar (BM) é a instituição responsável pelo planejamento e execução das atividades de policiamento ostensivo no Estado do Rio Grande do Sul (RS), assegurando o cumprimento da lei, a preservação da ordem pública, a incolumidade das pessoas e do patrimônio, bem como o exercício dos poderes constituídos (BRASIL, 1988; RIO GRANDE DO SUL, 1989; RIO GRANDE DO SUL, 1997). No cumprimento de sua missão constitucional, a corporação conta com o apoio do Departamento de Saúde (DS), responsável pelo planejamento, normatização, controle e execução das políticas e atividades de saúde física e mental dentro da instituição.

A Policlínica Odontológica Central (POC), subordinada ao DS e situada em Porto Alegre, RS, é a principal subunidade do Centro Médico-Odontológico da Brigada Militar (CMOBM), cuja missão é prestar assistência odontológica preventiva e curativa em clínica e outras especialidades, como cirurgia e traumatologia bucomaxilo-facial, dentística, endodontia, odontopediatria e periodontia. A POC executa a assistência odontológica especializada, possuindo a maior estrutura física e concentrando o maior volume de atendimentos prestados pelo CMOBM.

No final do ano de 2007, a BM e a Faculdade de Odontologia (FO) da Universidade Federal do Rio Grande do Sul (UFRGS) firmaram contrato de convênio para realização de estágio curricular não remunerado de alunos regularmente matriculados no Curso de Graduação da FO-UFRGS junto ao CMOBM. A Lei nº 11.788 , de 25 de setembro de 2008, define o estágio como o ato educativo escolar supervisionado, desenvolvido no ambiente de trabalho, que visa à preparação para o trabalho produtivo do estudante. $\mathrm{O}$ estágio integra o itinerário formativo do educando e faz parte do projeto pedagógico do curso.

O Estágio Supervisionado (ES) é a exteriorização do aprendizado acadêmico fora dos limites da Universidade. A ABENO define ES na Odontologia como "(...) o instrumento de integração e conhecimento do aluno com a realidade social e econômica de sua região e do trabalho de sua área. Ele deve, também, ser entendido como 0 atendimento integral ao paciente que 0 aluno de 
Odontologia presta à comunidade, intra e extramuros. $\mathrm{O}$ aluno pode cumpri-lo em atendimentos multidisciplinares e em serviços assistenciais públicos e privados". No ES, o discente é capaz de correlacionar a teoria e a prática, contribuindo para uma análise de pontos fortes e fracos das organizações e propondo melhorias para as instituições. O espaço destinado para o estágio faculta ao acadêmico a disponibilidade de consolidar seus conhecimentos com os entraves que somente a prática por meio do dia-a-dia pode oferecer. Nesta configuração, a troca de experiências faz com que o novo profissional torne-se mais preparado para atuar em diferentes áreas e lidar com a complexidade da realidade cotidiana.

As Diretrizes Curriculares Nacionais (DCN), de 2002, estipulam que $20 \%$ da carga horária do curso de graduação deva ser destinada à realização de estágios (BRASIL, 2006; HADDAD et al., 2006). Construir uma articulação entre as instituições de ensino e os serviços de saúde, que tenha como uma de suas competências ordenar a formação de recursos humanos em saúde tem sido um desafio permanente para os que fazem educação e saúde no Brasil (ARANTES et al., 2009).

O projeto político-pedagógico da FO-UFRGS refere que os dois últimos semestres do Curso de Odontologia devem ser destinados preferencialmente ao ES extramuros. Os estagiários do 9 e $10^{\circ}$ semestres têm 20 horas de atividades práticas semanais, além de 1 hora de Seminário de Integração. A supervisão desses estágios fica a cargo de professores das Disciplinas de Estagio Curricular Supervisionado I e II. Neste contexto, os campos de estágio têm sido ampliados e cuidadosamente avaliados e selecionados pela universidade. Dessa forma, o convênio de ES entre o CMOBM e a FO-UFRGS representa uma importante integração de interesses entre instituições públicas, onde acadêmicos de odontologia do último ano realizam atendimentos clínicos supervisionados nas dependências da POC, exercendo atividades preventivas e curativas em odontologia, devidamente orientados e supervisionados por cirurgiões-dentistas atuantes no serviço.

Os processos de avaliação de qualidade, desempenho e produtividade nos serviços de saúde (DONABEDIAN, 2003) representam uma ferramenta imprescindível nas práticas de gestão (BITTAR, 2001), particularmente em instituições públicas, ainda que, na cultura administrativa atual, estejam reduzidas a experiências pontuais. É preciso, inicialmente, reconhecer as dificuldades para medir a produtividade no setor saúde. Na medida em que existem diferentes formas de "outputs" produzidos por cada tipo de serviço de saúde, e que diferentes categorias profissionais podem estar envolvidas em cada atividade, é impossível expressar a produtividade em um único indicador (DAL POZ; PIERANTONI; VARELLA, 1997). Algumas estratégias para medir a produtividade nos serviços de saúde têm sido examinadas e exploradas (KALMAN; WITKOWISKI; OGAWA, 1992; KLERSY et al., 1995; MÉDICI; GIRARDI, 1996; SCHEFFLER; WAITZMAN; HILLMAN, 1996). No Brasil, algumas iniciativas têm sido desenvolvidas e documentadas (MOREIRA; TUMANG; OLIVEIRA, 1985; CHERCHIGLIA, 1994; BITTAR, 1996; MARINHO, 2003; PEREIRA et al., 2009). Outros estudos, ainda que pouco freqüentes, têm se dedicado a avaliar especificamente a produtividade de estudantes de odontologia (HOLMES et al., 2000; BEAN et al., 2007; HOLMES et al., 2009; BERG et al., 2010).

Outras Polícias Militares do Brasil possuem serviços odontológicos semelhantes aos encontrados na BM. A cobertura do Serviço Odontológico da Polícia Militar da Bahia foi avaliada no estudo de Ribeiro-Sobrinho et al. (2008). Os autores encontraram uma adequada cobertura potencial de recursos humanos (1 cirurgião-dentista/1.618 habitantes) e uma taxa de cobertura real abaixo do padrão proposto pelo Ministério da Saúde $(0,39$ procedimento/habitante/ano). A baixa cobertura real encontrada, segundo os autores, poderia estar relacionada à baixa produtividade do serviço, cujas razões sugeridas foram problemas estruturais do serviço e a falta de um sistema de gestão do desempenho da qualidade da prática profissional com metas a serem cumpridas.
$\mathrm{Na}$ perspectiva de avaliação de serviços de saúde, pressupõe-se que a implantação do ES na POC seja uma forma de ampliar a capacidade de atendimento, a partir da ampliação de recursos humanos - os estagiários -, contribuindo para minimizar o déficit de cobertura potencial e aumentar o desempenho do serviço. Este pressuposto, entretanto, carece de confirmações baseadas em pesquisa. Desse modo, faz-se pertinente quantificar o incremento de produtividade do serviço da POC após a implementação do ES. Assim, o objetivo do presente estudo foi analisar o impacto do ES de alunos da FO-UFRGS, com enfoque nos resultados, no desempenho da POC da BM no período de abril de 2008 a dezembro de 2009.

\section{Metodologia}

Este estudo seguiu delineamento descritivo e analítico de séries históricas, com estrutura de abordagem quantitativa, envolvendo o levantamento do número de pacientes atendidos (PA) e procedimentos odontológicos (PO) realizados, de forma a apresentar as médias mensais de produtividade, sistematizada segundo a categoria - estagiários (E) e cirurgiões-dentistas (CD) -, seguida de comparação da equivalência de produtividade entre as categorias estudadas.

O levantamento de dados foi realizado na Policlínica Odontológica Central (POC) e na Seção Técnico Administrativa (STAdm) do Centro Médico-Odontológico da Brigada Militar (CMOBM). Já a análise dos dados foi conduzida na Academia de Polícia Militar (APM) da BM, em Porto Alegre - RS.

A população em estudo foi composta por todos os $E$ e CD que atuaram na POC, durante um período de 21 meses, entre abril de 2008 e dezembro de 2009. O número total de CD que atuaram no serviço da POC (total do efetivo clínico) no período estudado foi de $14 \mathrm{CD}$, entre militares e civis. Entretanto, devido a diversos tipos de afastamentos funcionais (cursos, férias, licenças, convocações, etc.), o número mensal de CD que atuaram no serviço foi variável, e esta variação foi considerada no estudo.

Já o número total de $\mathrm{E}$ que atuaram no período foi regular, dentro de cada semestre, com a atuação de $2 \mathrm{E}$ por turno de trabalho (2 $\mathrm{E}$ manhã, $2 \mathrm{E}$ tarde), durante 9 turnos semanais. Entretanto, a carga horária individual dos $E$ era variável, ou seja, alguns alunos estagiaram três turnos, outros dois e outros apenas um turno, dentro de cada semestre letivo. No período estudado, realizaram ES na POC um total de 51 (cinqüenta e um) E, em média mais de 12 alunos por semestre. Para o presente estudo não foi analisada a produtividade individual dos $E$, mas sim a produtividade geral do ES, a partir das somas de produtividade individual de todos os E. Cabe ressaltar que nos meses de julho de 2008 e julho de 2009, os E não atuaram no serviço devido ao período de férias escolares.

A variável PA quantifica 0 volume de atendimentos realizados, contabilizando o número de consultas odontológicas realizadas. Cumpre salientar que nos casos em que, por exemplo, um mesmo paciente realizou mais de uma consulta dentro de um mesmo mês, todas estas consultas foram computadas para fins de quantificação de PA naquele mês.

Já a variável $P O$ quantifica a densidade dos atendimentos realizados, registrando o número de procedimentos odontológicos realizados. A classificação destes procedimentos seguiu as rotinas internas já estabelecidas pela STAdm do CMOBM. Neste particular, procedimentos clínicos mais complexos levam a uma maior quantificação do número final de PO; como exemplos, citamos três situações clínicas: a primeira, em que é realizada uma restauração provisória mésio-ocluso-distal, gerando apenas um PO (restaurações provisórias são computadas por dente); a segunda, em que é realizada uma restauração definitiva de resina composta mésio-ocluso-distal, sob isolamento absoluto do campo operatório, gerando $4 \mathrm{PO}$ (3 PO referentes à restauração definitiva, que é computada por faces restauradas, e $1 \mathrm{PO}$ referente ao isolamento absoluto); e a terceira, em que é realizada uma RAP (raspagem, alisamento e polimento) das arcadas superior e inferior, seguida de 
instrução de higiene bucal (IHB) e 2 radiografias periapicais, gerando $5 \mathrm{PO}(1 \mathrm{PO}$ referente à RAP da arcada superior, $1 \mathrm{PO}$ referente à RAP da arcada inferior, $1 \mathrm{PO}$ referente à IHB, $2 \mathrm{PO}$ referentes aos dois exames radiográficos periapicais).

Inicialmente, foi realizado um levantamento mensal do número total de $\mathrm{PA}$ e de $\mathrm{PO}$ realizados por $\mathrm{E}$ e $\mathrm{CD}$, de acordo com os registros existentes nos mapas mensais de produtividade individual da STAdm do CMOBM. Estes dados foram registrados em uma planilha de registro de dados (PRD).

A seguir, a partir da razão entre o número total mensal de PA por todos os $C D$ e o número total de $C D$ que atuaram no mês, foi calculada a média de PA por CD em cada mês. Da mesma forma, utilizando a mesma estratégia de cálculo, foi encontrada a média mensal de PO realizados por $C D$. Estes dados também foram registrados na $P R D$.

A seguir, foi realizada a comparação da equivalência de produtividade entre $E$ e $C D$, calculada mensalmente, a partir da proposta de criação de um Indicador de Produtividade do Estágio (IPE). O cálculo do IPE foi realizado a partir da razão entre o número total de $\mathrm{PA}$ por $\mathrm{E}$ e a média de $\mathrm{PA}$ por $\mathrm{CD}$, bem como a partir da razão entre o número total de $\mathrm{PO}$ realizados por $\mathrm{E}$ e a média de $\mathrm{PO}$ realizados por CD. Os valores obtidos a partir da comparação da equivalência de produtividade expressam, dessa forma, o indicador de produtividade mensal de todos os estagiários em termos de incremento na produtividade geral do corpo clínico da POC, ou, em outras palavras, respondem à questão: "quantos $\mathrm{CD}$ a POC obteve a mais, em termos de produtividade ( $\mathrm{PA}+\mathrm{PO})$ com a atuação de todos os estagiários?". A este valor foi atribuída a denominação IPE.

Os valores mensais de IPE foram calculados, conforme já descrito, relativamente ao número de $\mathrm{PA}$ (IPE-pa) e ao número de $P O$ realizados (IPE-po). Finalmente, o IPE geral (IPE-g) mensal fo calculado através da média mensal entre o IPE-pa e o IPE-po (Figura 1).

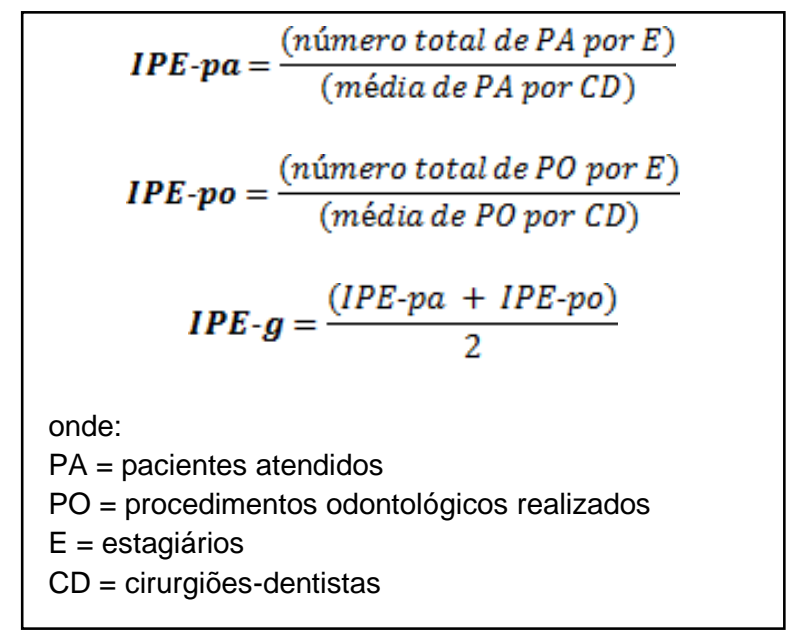

Figura 1. Fórmulas de IPE-pa, IPE-po e IPE-g.

Os valores médios gerais de IPE-pa, IPE-po e IPE-g também foram calculados, através das médias gerais de cada indicador entre todos os meses em estudo. O número médio geral de PO realizados por atendimento também foi calculado, tanto para $C D$ quanto para $E$. Finalmente, foi realizado o cálculo de incremento percentual na produtividade do serviço, a partir da produtividade de $C D$ e $E$. Os resultados foram expressos através de tabelas e gráficos, utilizando estatísticas descritivas simples (freqüências, médias e desvio-padrão).

Este estudo foi encaminhado, previamente a sua realização, ao Instituto de Pesquisa da Brigada Militar (IPBM), tendo sido registrado e aprovado sob o n. ${ }^{\circ}$ 2839/2010.

\section{Resultados}

A Tabela 1 apresenta a distribuição mensal do número de PA e PO realizados por CD e E no período estudado, bem como o número mensal de CD que atuaram na $P O C$ e as médias mensais de PA e PO por CD. Esta tabela também expressa os valores de IPE-pa, IPE-po e IPE-g ao longo de todos os meses investigados no estudo, bem como os valores médios gerais e desvios-padrão no período.

O número médio de PA por $\mathrm{CD}$ ao longo do período foi de 86,7 pacientes, enquanto que o número médio de $\mathrm{PA}$ por $\mathrm{E}$ foi de 112,7 , com conseqüente IPE-pa médio de 1,63, variando de 0,15 a 3,65 . Isto representa um acréscimo de produtividade médio de 1,63 $C D$ na POC, com a implantação do $E S$, em relação ao numero de $\mathrm{PA}$. Similarmente, o número médio de $\mathrm{PO}$ realizados por $\mathrm{CD}$ no período foi de 315,2 , enquanto que o número médio de $\mathrm{PO}$ realizados por $E$ foi de 454,3 , com conseqüente IPE-po médio de 1,48 , variando de 0,49 a 2,69. Isto representa um acréscimo de produtividade médio de 1,48 CD na POC, com a implantação do $\mathrm{ES}$, em relação ao numero de $\mathrm{PO}$.

Quanto ao IPE-g, que traduz a média entre IPE-pa e IPEpo, obteve-se um valor médio de 1,55 , com variação de 0,32 a 3,17 , significando que a existência do ES trouxe um acréscimo de produtividade geral de 1,55 CD para o serviço de odontologia da POC no período entre abril de 2008 e dezembro de 2009.

$\mathrm{Na}$ Tabela 2, é possível observar o número total de PA e $P O$ realizados por $C D$ e $E$, bem como médias de $P O$ realizados por $\mathrm{CD}$ e $\mathrm{E}$ por atendimento, o incremento percentual de PA e PO realizados por $E$ frente ao total de $P A$ e $P O$ realizados, além do incremento percentual médio na produtividade do serviço no período estudado. Destaca-se, neste aspecto, que 0 ES representou um incremento de produtividade médio de $9,94 \%$ para PA e de $10,97 \%$ para PO realizados, numa média geral de incremento de produtividade do serviço de $10,46 \%$.

Tabela 2. Número total de pacientes atendidos (PA) e procedimentos odontológicos (PO) realizados por cirurgiõesdentistas (CD) e estagiários (E), bem como médias de $P O$ realizados por $C D$ e $E$ por atendimento, incremento percentual de $\mathrm{PA}$ e $\mathrm{PO}$ realizados por $\mathrm{E}$ frente ao total de $\mathrm{PA}$ e $\mathrm{PO}$ realizados por $C D$ e incremento percentual médio na produtividade geral do serviço no período de abr/08 a dez/09.

\begin{tabular}{ccccc}
\hline & PA (total) & PO (total) & $\begin{array}{c}\text { Média PO } \\
\text { por } \\
\text { atendimento }\end{array}$ & Média \\
\hline CD & 21541 & 78659 & 3,65 & - \\
E & 2142 & 8632 & 4,03 & -
\end{tabular}

$\begin{array}{ccccc}\text { Incremento } & 9,94 & 10,97 & - & 10,46\end{array}$

Na Figura 2 é possível observar, graficamente, a evolução das variações de IPE-pa, IPE-po e IPE-g ao longo do período estudado, com destaque para os valores mínimos e máximos, representados pelos meses de agosto de 2009 e abril de 2008, respectivamente. 
Tabela 1. Distribuição mensal do número de cirurgiões-dentistas (CD) atuantes, pacientes atendidos (PA) e procedimentos odontológicos (PO) realizados por $C D$ e estagiários (E), valores mensais de IPE-pa, IPE-po e IPE-g, bem como médias gerais e desvio padrão.

\begin{tabular}{|c|c|c|c|c|c|c|c|c|c|c|}
\hline & № $C D$ & $\begin{array}{c}\text { Total PA } \\
\text { (CD) }\end{array}$ & $\begin{array}{l}\text { Média } \\
\text { PA/CD }\end{array}$ & $\begin{array}{c}\text { Total PA } \\
\text { (E) }\end{array}$ & IPE-pa & $\begin{array}{c}\text { Total PO } \\
\text { (CD) }\end{array}$ & $\begin{array}{c}\text { Média } \\
\text { PO/CD }\end{array}$ & $\begin{array}{c}\text { Total PO } \\
\text { (E) }\end{array}$ & IPE-po & IPE-g \\
\hline$a b r / 08$ & 12 & 750 & 62,50 & 228 & 3,65 & 3428 & 285,67 & 769 & 2,69 & 3,17 \\
\hline mai/08 & 12 & 940 & 78,33 & 160 & 2.04 & 3895 & 324,58 & 583 & 1,80 & 1,92 \\
\hline jun/08 & 12 & 791 & 65,92 & 92 & 1,40 & 3797 & 316,42 & 676 & 2,14 & 1,77 \\
\hline jul/08 & 12 & 943 & 78,58 & - & - & 4136 & 344,67 & - & - & - \\
\hline ago/08 & 12 & 867 & 72,25 & 60 & 0,83 & 3821 & 318,42 & 604 & 1,90 & 1,36 \\
\hline set/08 & 13 & 922 & 70,92 & 122 & 1,72 & 4164 & 320,31 & 399 & 1,25 & 1,48 \\
\hline out/08 & 13 & 885 & 68,08 & 106 & 1,56 & 4247 & 326,69 & 416 & 1,27 & 1,42 \\
\hline nov/08 & 13 & 782 & 60,15 & 131 & 2,18 & 3515 & 270,38 & 490 & 1,81 & 1,99 \\
\hline $\mathrm{dez} / 08$ & 12 & 658 & 54,83 & 79 & 1,44 & 2970 & 247,50 & 310 & 1,25 & 1,35 \\
\hline $\mathrm{jan} / 09$ & 14 & 626 & 44,71 & 67 & 1,50 & 3032 & 216,57 & 224 & 1,03 & 1,27 \\
\hline $\mathrm{fev} / 09$ & 12 & 440 & 36,67 & 80 & 2,18 & 2125 & 177,08 & 328 & 1,85 & 2,02 \\
\hline $\mathrm{mar} / 09$ & 11 & 664 & 60,36 & 93 & 1,54 & 3322 & 302,00 & 391 & 1,29 & 1,42 \\
\hline $\mathrm{abr} / 09$ & 12 & 786 & 65,50 & 157 & 2,40 & 3788 & 315,67 & 387 & 1,23 & 1,81 \\
\hline mai/09 & 11 & 1688 & 153,45 & 204 & 1,33 & 4547 & 413,36 & 668 & 1,62 & 1,47 \\
\hline jun/09 & 11 & 2154 & 195,82 & 108 & 0,55 & 5104 & 464,00 & 469 & 1,01 & 0,78 \\
\hline jul/09 & 12 & 880 & 73,33 & - & - & 4521 & 376,75 & - & - & - \\
\hline ago/09 & 12 & 3955 & 329,58 & 48 & 0,15 & 3955 & 329,58 & 160 & 0,49 & 0,32 \\
\hline set/09 & 12 & 725 & 60,42 & 124 & 2,05 & 3749 & 312,42 & 497 & 1,59 & 1,82 \\
\hline out/09 & 11 & 728 & 66,18 & 135 & 2,04 & 3777 & 343,36 & 588 & 1,71 & 1,88 \\
\hline nov/09 & 11 & 756 & 68,73 & 91 & 1,32 & 3500 & 318,18 & 432 & 1,36 & 1,34 \\
\hline dez/09 & 11 & 601 & 54,64 & 57 & 1,04 & 3266 & 296,91 & 241 & 0,81 & 0,93 \\
\hline Média & 11,95 & 1025,76 & 86,71 & 112,74 & 1,63 & 3745,67 & 315,26 & 454,32 & 1,48 & 1,55 \\
\hline D.P. & 0,79 & 750,64 & 64,40 & 47,35 & 0,74 & 621,28 & 59,56 & 160,51 & 0,49 & 0,57 \\
\hline
\end{tabular}

$\left.{ }^{*}\right)$ meses de jul/08 e jul/09: período de férias de estagiários.

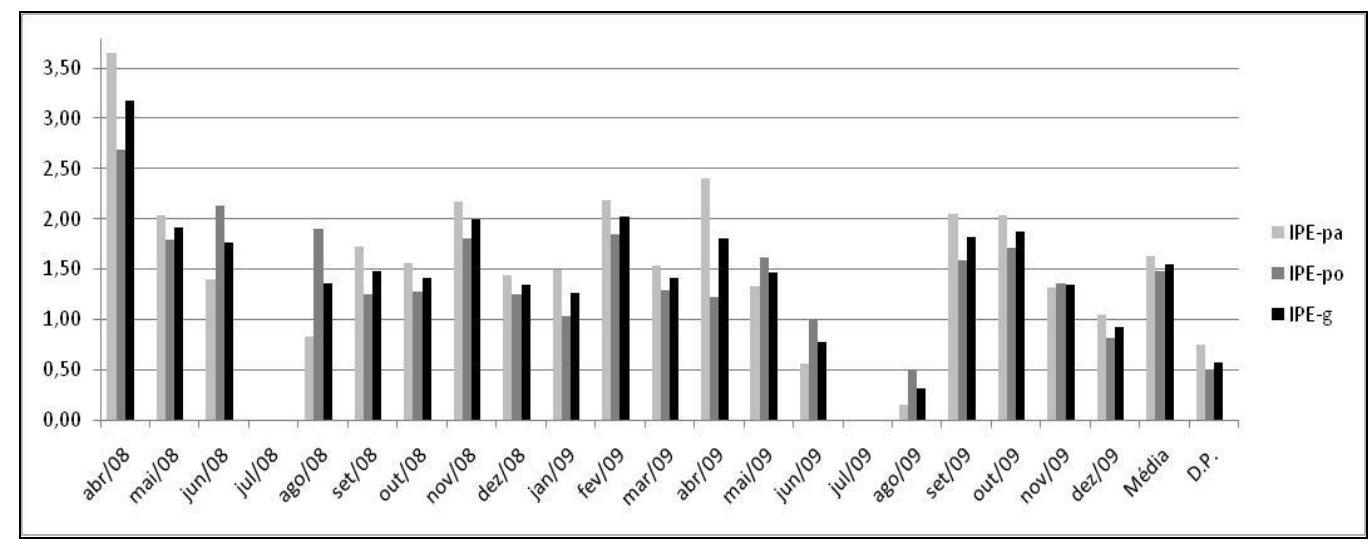

Figura 2. Gráfico de distribuição dos valores mensais de IPE-pa, IPE-po e IPE-g, bem como média geral e desvio-padrão dos indicadores no período estudado. ${ }^{*}$ ) meses de jul/08 e jul/09: período de férias de estagiários.

\section{Discussão}

O ES é um momento primordial na graduação, permitindo ao aluno o contato com a profissão, inserindo-o em um cenário capaz de mesclar situações práticas, técnicas, científicas e sócioculturais, contribuindo assim na integração da aprendizagem acadêmica com o contexto profissional. Uma das maneiras das faculdades incorporarem as novas deliberações das DCN é o ES, cujos objetivos são fomentar a relação ensino-serviços, ampliar as relações da universidade com a sociedade e colocar o futuro profissional em contato com as diversas realidades. (RODRIGUES, 1995; RODRIGUES; LEITÃO, 2000). Neste particular, o ES de alunos da FO-UFRGS junto à $P O C$ da BM parece estar cumprindo sua proposta, mostrando-se interessante para ambas as instituições públicas. A literatura, entretanto, é carente de estudos na área odontológica capazes de embasar, quantitativa e qualitativamente, o incentivo às práticas de parcerias institucionais para realização de ES (ARANTES et al., 2009).
O convênio de ES que existe entre a BM e a UFRGS é caracterizado pela cooperação entre as instituições, sem envolver qualquer transferência de recursos financeiros. Assim, sob a ótica de gestão, em termos específicos de produtividade, o ES demonstrou ter alta eficiência, já que foi capaz de gerar indicadores de produtividade relevantes, elevando em mais de $10 \%$ a produtividade geral do serviço, sem despender nenhum custo relativo aos recursos humanos adicionais (os estagiários). Dessa forma, o impacto do ES sobre o desempenho da POC, de acordo com os resultados obtidos neste estudo, pode ser considerado significativo sob o prisma de resultados, já que a implementação do ES representou um ganho de produtividade médio equivalente à força de trabalho de aproximadamente 1,5 CD (IPE-g = 1,55).

Ao longo do período estudado, houve variações importantes nos indicadores mensais de IPE-g. É possível observar que os maiores valores de IPE-g ocorreram no mês de abril de 2008. Isto se deu, possivelmente, devido à conjunção de alguns fatores: (1) este foi o primeiro mês de implementação do estágio na $\mathrm{POC}$, quando as rotinas de trabalho dos estagiários junto ao 
serviço ainda passavam por adaptações, e houve uma sobrecarga no volume de encaminhamentos para estagiários - o que foi revisto já no mês subseqüente, a fim de oferecer melhores condições de trabalho para estagiários e preceptores; (2) neste mês, as médias de PA $(62,5)$ e PO $(285,7)$ realizados por CD ficaram abaixo das médias gerais de PA $(86,7)$ e PO $(315,7)$ realizados no período estudado e, considerando que estes valores representam o denominador da equação de cálculo de IPE-g, esta redução trouxe aumento dos valores do indicador. Já o mês de agosto de 2009 apresentou os menores valores de IPE-g, certamente devido à realização de juntas de inspeção de saúde (exames para seleção de ingresso na carreira militar), que elevaram consideravelmente a média de PA por $C D$, além da redução da carga horária mensal de trabalho dos estagiários, uma vez que o semestre letivo iniciou apenas na metade do referido mês.

Outro resultado que merece discussão é a diferença encontrada entre 0 número médio de $\mathrm{PO}$ por atendimento realizados por $E(4,0)$ e $C D(3,6)$. Observa-se que os $E$ realizam, em média, um número maior de $\mathrm{PO}$ em cada atendimento do que os CD. Esta diferença é explicada, possivelmente, pelo fator tempo: os $E$ realizam atendimentos mais longos do que os $C D$, tendo assim a oportunidade de realizar uma maior quantidade de $\mathrm{PO}$ em uma mesma consulta; já os CD atendem um maior volume (PA) de pacientes, o que reduz o tempo clínico de cada atendimento, reduzindo também a média de $\mathrm{PO}$ em cada atendimento.

Relativamente aos indicadores de produtividade propostos neste estudo (IPE-pa, IPE-po e IPE-g), são necessárias algumas considerações: (1) é preciso mencionar que são indicadores baseados no número de $\mathrm{PA}$ e $\mathrm{PO}$ realizados por $\mathrm{E}$ e $C D$, registros estes rotineiramente empregados nos controles mensais da STAdm do CMOBM, sendo portanto, usuais e de fácil acesso; (2) estes indicadores são representativos do volume (IPEpa) e da densidade (IPE-po) dos atendimentos realizados por estagiários no serviço - volume e densidade de atendimentos são dados importantes para a gestão de qualquer serviço odontológico; (3) estes indicadores possuem aplicabilidade, pois refletem o impacto do ES sobre o serviço, em termos de desempenho e produtividade, a partir de uma equalização de CD e E com base na carga horária; (4) estes indicadores podem ser reproduzidos e utilizados em novos estudos acerca do tema, tanto na POC da BM quanto em qualquer outro local onde seja implantado um ES em odontologia; desse modo, a utilização destes indicadores possibilita a realização de brenchmarking, ou seja, comparações de desempenho e produtividade do ES, por exemplo, entre diferentes períodos de tempo em um mesmo serviço, entre diferentes turmas de uma mesma universidade, entre diversos serviços de odontologia onde há ES, etc.

A avaliação de serviços de saúde em geral apresenta dificuldades que têm sido amplamente comentadas e discutidas na literatura. No Brasil, a avaliação de profissionais e de serviços tem sido recomendada com base nos indicadores mais facilmente obteníveis, a maioria deles indicadores de processo, mas alguns também de resultados, especialmente os indicativos de produção e produtividade (BITTAR, 2001), a exemplo dos indicadores usados no presente estudo. No estudo de Marinho (2003), que realizou uma avaliação dos serviços ambulatoriais e hospitalares no estado do Rio de Janeiro, foi analisada a capacidade de atendimento e o total de procedimentos ambulatoriais per capita, à semelhança da variável PO do presente estudo. Entretanto, a avaliação de Marinho (2003) criou escores de eficiência, utilizando um modelo de regressão que levou em conta diversos outros fatores não considerados na presente investigação.

Outros estudos que analisaram a produtividade de estudantes de odontologia (MOREIRA; TUMANG; OLIVEIRA, 1985; HOLMES et al., 2000; BEAN et al., 2007; HOLMES et al., 2009; BERG et al., 2010) também consideraram, assim como no presente estudo, o número de consultas e de procedimentos realizados pelos alunos; entretanto, não foi considerada a produtividade dos $C D$ dos diferentes serviços, impedindo qualquer tentativa de comparação, em termos de impacto do ES sobre a produtividade do serviço, entre os estudos. Percebe-se, assim, a dificuldade de se trabalhar com generalizações quando se trata de mensurar produtividade em serviços de saúde. A comparação entre unidades de serviço é impedida pela diversidade de atores ou componentes internos e externos à organização (DAL POZ; PIERANTONI; VARELLA, 1997). Neste sentido, a utilização dos indicadores de produtividade aqui propostos visa contribuir para a possibilidade de comparações entre futuras investigações.

$O$ presente estudo possui, certamente, limitações. O período de acompanhamento de 21 meses é considerado ainda reduzido para uma observação segura dos indicadores propostos; novos estudos seguindo esta mesma metodologia devem ser realizados ao longo do tempo na POC. Além disso, este estudo verificou apenas o impacto do ES sobre o desempenho da POC; é preciso considerar, entretanto, que este local não é representativo de todo o serviço de odontologia da BM. Outro aspecto que traz limitação diz respeito à validade externa: o impacto do ES sobre outros serviços de odontologia que também mantém convênios com a FO-UFRGS não foi testado; sugere-se, portanto, que outros estudos com metodologia similar sejam conduzidos nestes outros locais de estágio.

Finalmente, é preciso referir que embora o presente estudo tenha focado a análise do desempenho do serviço a partir de indicadores quantitativos de produtividade, existem também outros importantes fatores acerca da implementação do ES na POC que não foram estudados. Aspectos qualitativos das interações entre os estagiários, a equipe de trabalho, os pacientes, as chefias, os preceptores e os professores (CAVACA et al., 2010), bem como inúmeros fatores relativos ao processo de ensino-aprendizado extra-muros à FO-UFRGS, particularmente em um ambiente militar como a POC da BM, além de observações acerca da qualidade do serviço, antes a após a implementação do ES, entre outros, são enfoques que merecem atenção e novos estudos devem ser desenvolvidos na área.

\section{Conclusões}

Os resultados deste trabalho permitiram concluir que: (1) - ES proporcionou acréscimo de produtividade equivalente a mais de um e meio $(1,5)$ CD por mês para o serviço da POC da BM; (2) a implantação do ES trouxe um aumento médio mensal de 10,46\% na produtividade geral do serviço de odontologia da POC da BM.

Os indicadores propostos neste estudo (IPE-pa, IPE-po e IPE-g) representam ferramentas interessantes para monitoramento do impacto da implementação do ES sobre o desempenho de serviços odontológicos, com enfoque nos resultados. Ainda assim, são necessários estudos com outros enfoques acerca do tema, envolvendo também indicadores qualitativos, a fim de integrar e correlacionar avaliações de qualidade, desempenho e produtividade do serviço com os aspectos pedagógicos de ensinoaprendizagem relativos ao ES no ambiente de trabalho.

\section{Referências}

ARANTES, A.C.C. et al. Estágio supervisionado: qual a sua contribuição para a formação do cirurgião-dentista de acordo com as diretrizes curriculares nacionais? R. APS, Juiz de Fora, v. 12, n. 2, p. 150-160, abr./jun. 2009.

ASSOCIAÇÃO BRASILEIRA DE ENSINO ODONTOLÓGICO. Estágios supervisionados. Reunião paralela da ABENO. 2003. Disponível em: <http://www.abeno.org.br>. Acesso em: $15 \mathrm{fev}$. 2010.

BEAN, C.Y. et al. Comparing fourth-year dental student productivity and experiences in a dental school with community-based clinical education. J. Dent. Educ., Washington, v. 71, no. 8, p. 1020-1026, Aug. 2007. 
BERG, R. et al. Impact of the University of Colorado's Advanced Clinical Training and Service (ACTS) program on dental students' clinical experience and cognitive skills, 1994-2006. J. Dent. Educ., Washington, v. 74, no. 4, p. 423-433, Apr. 2010.

BITTAR, O. J. N. V. Indicadores de qualidade e quantidade em saúde. R. Adm. Saúde, São Paulo, v. 3, n.12, p. 21-28, jul./set. 2001

Produtividade em hospitais de acordo com alguns indicadores hospitalares. R. Saúde Públ., Rio de Janeiro, v. 30, n.1, p. 53-60, fev. 1996.

BRASIL. Constituição (1988). Constituição da República Federativa do Brasil: promulgada em 5 de outubro de 1988. Brasília, 1988.

Lei no 11.788 , de 25 de setembro de 2008. Dispõe sobre o estágio de estudantes... Diário Oficial [da] República Federativa do Brasil, Brasília, DF, 26 set. 2008.

Ministério da Saúde. A aderência dos cursos de graduação em enfermagem, medicina e odontologia às diretrizes curriculares nacionais. Brasília, 2006.

CAVACA, A.G. et al. A relação professor-aluno no ensino da odontologia na Universidade Federal do Espírito Santo. Trab. Educ. Saúde, Rio de Janeiro, v. 8 n. 2, p. 305-318, jul./out. 2010.

CHERCHIGLIA, M.L. Remuneração do trabalho médico: um estudo sobre seus sistemas e formas em hospitais gerais de Belo Horizonte. Cad. Saúde Públ., Rio de Janeiro, v. 10, n. 1, p. 67-79, 1994.

DAL POZ, M.R; PIERANTONI, C.R.; VARELLA, T.C. Produtividade e desempenho dos recursos humanos nos serviços de saúde. [S.I.]: Organização Pan-americana de Saúde, jun. 1997. Disponível em: <http://www.paho.org/Portuguese/HSP/HSR/HSR01/produtividadee-desempenho.pdf>. Acesso em: 02 set. 2010.

DONABEDIAN, A. An introduction to quality assurance in health care. New York: Oxford University Press, 2003. 200 p.

HADDAD, A. E. et al. A trajetória dos cursos de graduação na área da saúde: 1991-2004. Brasília: Instituto Nacional de Estudos e Pesquisas Educacionais Anísio Teixeira, 2006.

HOLMES, D. C. et al. Student productivity in a comprehensive care program without numeric requirements. J. Dent. Educ., Washington, v. 64, no. 11, p. 745-754, Nov. 2000.

HOLMES, D.C. et al. Comparison of student productivity in fourhanded clinic and regular unassisted clinic. J. Dent. Educ., Washington, v. 73, no. 9, p.1083-1089, Sept. 2009.

KALMAN, M.K.; WITKOWISKI, D.E.; OGAWA, G.S. Increasing pharmacy productivity by expanding the role of pharmacy technicians. AJHP: Am. J. Health Syst. Pharm., Bethesda, MD, v. 49, p. 84-89, Jan. 1992.

KLERSY, C. et al. A measure of productivity in cardiology based on medical acts. G. Ital. Cardiol., Pádua, v. 25, no. 4, p. 433-443, Apr. 1995.

MARINHO, A. Avaliação da eficiência técnica nos serviços de saúde nos municípios do estado do Rio de Janeiro. RBE, Rio de Janeiro, v. 57, n. 2, p. 515-534, jul./set. 2003.
MÉDICI, A.C.; GIRARDI, S.N. Emprego, remuneração de pessoal e produtividade em saúde: um balanço da literatura recente. Divulg. Saúde Debate, Londrina, v. 14, p. 38-43, ago. 1996.

MOREIRA, B.H.W.; TUMANG, A.J.; OLIVEIRA, S.P. Participaçao de estudantes de odontologia em programas de integração docente-assistencial. R. Bras. Odontol., Rio de Janeiro, v. 42, n. 4, p. 30-36, jul./ago. 1985.

PEREIRA, C.R.S. et al. Impacto da Estratégia Saúde da Família com equipe de saúde bucal sobre a utilização de serviços odontológicos. Cad. Saúde Pública, Rio de Janeiro, v. 25, n. 5, p. 985-996, maio 2009

RIBEIRO-SOBRINHO, C. et al. Avaliação da cobertura do Serviço Odontológico da Polícia Militar da Bahia em Salvador, Bahia, Brasil. Cad. Saúde Pública, Rio de Janeiro, v. 24, n. 2, p. 295-302, fev. 2008.

RIO GRANDE DO SUL. Constituição (1989). Constituição do estado do Rio Grande do Sul: texto constitucional de 3 de outubro de 1989. Porto Alegre, 1989.

Lei Complementar no 10.991, de 18 de agosto de 1997. Dispõe sobre a Organização Básica da Brigada Militar do Estado e dá outras providências. Estatuto dos Servidores Militares da Brigada Militar do RS. Porto Alegre: Corag, 2001.

RODRIGUES, E.N. Primeiro estágio curricular: relato de experiência. R. Bras. Enferm., Brasília, v. 48, n. 4, p. 436-443, out./dez. 1995.

RODRIGUES, M.S.P.; LEITÃO, G.C.M. Estágio curricular supervisionado com ênfase no desenvolvimento da autonomia e da responsabilidade. Texto \& Contexto Enferm., Florianópolis, v. 9, n 3, p. 216-229, ago./dez. 2000.

SCHEFFLER, R.M.; WAITZMAN, N.J.; HILLMAN, J.M. The productivity of physician assistants and nurse practitioners and health work force policy in the era of managed health care. J. Allied Health., Thorofare, NJ, v. 25, no. 3, p. 207-217, Summer 1996.

UNIVERSIDADE FEDERAL DO RIO GRANDE DO SUL. Faculdade de Odontologia. Projeto político pedagógico: 2005/1. Disponível em: <http://www.ufrgs.br/odonto/projeto_politico.pdf>. Acesso em: 02 set. 2010. 